\title{
Rising Incidence of Metallo B-Lacatamse Producers in Burns Injury: A Ticking Time Bomb
}

\author{
Sumaira Qayoom Beigh, G.P. Aaftab, M.S. Rashmi* and Muzafar Amin \\ Department of Microbiology, VIMS, Ballari, Karnataka-580025, India \\ *Corresponding author
}

\begin{tabular}{|l|}
\hline K e y w o r d s \\
Pseudomonas \\
aeruginosa, Metallo \\
Beta Lactamases \\
(MBLs), Combined \\
Disc Test (CDT), \\
Imipenem -EDTA \\
Double disc \\
Synergy Test \\
(DDST) \\
\hline Article Info \\
\hline Accepted: \\
15 March 2019 \\
Available Online: \\
10 April 2019
\end{tabular}

\section{Introduction}

The extensively burned patient is at increased risk of wound infection as well as infection of other organs as a result of various degrees of impairment of host defense mechanism.

Infection of burns is common because the
One of the common causes of morbidity and mortality following burn injury is infections. The emergence of metallo- $\beta$-lactamase (MBL) in Gram negative bacilli (GNB) has reduced the available therapeutic options for effective treatment. Therefore, for screening of carbapenemase producers, a simple and inexpensive testing method is essential. To know the prevalence of MBL production in various gram negative isolates, to evaluate different phenotypic methods to detect $\mathrm{MBL}$ production and to find out antibiotic sensitivity profile of MBL producing gram negative bacilli. Wound swabs taken from about 100 patients admitted in the burns ward were processed. Kirby Bauer Disc diffusion test was performed on all isolates for antimicrobial susceptibility. Imipenem resistant clinical isolates were taken as positive for MBL screening. Two different methods using EDTA as MBL inhibitor were evaluated: (i) Combined disk synergy test with imipenem (CDST-IPM), (ii) Double-disk synergy test with imipenem (DDST-IPM), according to CLSI guidelines. The most common isolate was Pseudomonas aeruginosa (23\%), followed by Staphylococcus aureus (22\%), Escherichia coli (14\%), Klebsiella pneumoniae (9\%), Klebsiella oxytoca (8\%),CONS(7\%), Proteus mirabilis (5\%), Citrobacterfreundii (4\%), Citrobacter koseri (3\%) and Acinetobacterbaumanii (2\%).Out of 82 Gram negative isolates, 17 were resistant to imipenem and were considered screening positive and further tested for MBL production out of which only10 isolates were confirmed as MBL producers. A.baumanii was the predominant MBL producer followed by P.aeruginosa. MRSA rate among S.aureus was $56 \%$, but all were susceptible to glycopeptides and oxazolidinone. Early detection of MBL-carrying organisms, is of paramount clinical importance, as it allows rapid initiation of strict infection control practices as well as therapeutic guidance for confirmed infection. 
particularly nosocomial isolates, limits the available therapeutic options for effective treatment of burn wound infections.

The introduction of carbapenem into clinical practice represented a great advancement for the treatment of $\beta$-lactam resistant bacteria. Due to their broad spectrum of activity and stability to hydrolysis by most $\beta$-lactamase, the carbapenems have been the drugs of choice for treatment of infections caused by penicillin or cephalosporin resistant gram negative bacilli. However, the clinical utility of carbapenems is under threat with the emergence of acquired carbapenemases, particularly Ambler class B metalloBlactamases (MBLs). Carbapenemases may be definedas beta-lactamases that significantly hydrolyze at least imipenem or meropenem. Carbapenemases involved in acquired resistance are of Ambler molecular classes A, Band D. Class B or the metalloenzymes (MBL) are the most significant carbapenemases $^{[2]}$ The metallobetalactamases (MBLs)belong to group B and are enzymes requiring divalent cations as cofactors for enzyme activity, being inhibited by the action of a metal ion chelator ${ }^{3}$. Such enzymes have emerged in many geographical locations and often confer high-level resistance to all Blactams except aztreonam. Five enzyme types have been identified (IMP, VIM, SPM, GIM, and SIM types), involving various host organisms, most commonly Pseudomonas spp., Acinetobacter spp., and Enterobacteriaceae.

A particular concern is that acquired MBL genes are located on integron structures that reside on mobile genetic elements such as plasmids or transposons, thus enabling widespread dissemination. Clinical infections with such organisms pose serious therapeutic challenges, with increasing reports of poor patient outcomes and death ${ }^{4}$.These isolates have been responsible for serious infections such as septicemia and pneumonia and have been associated with failure of therapy with carbapenems. These organisms can be transmitted easily from one patient to another. Thus, outbreaks are not uncommon in burn units $^{[5]}$ Therefore, detection of MBL producing gram negative bacilli especially $P$. aeruginosa is crucial for optimal treatment of patients particularly critically ill and hospitalized patients and to control the spread of resistance. The present study was planned to find out the prevalence of MBL producing bacteria and to optimize the therapeutic use of antimicrobials for treating metallo-betalactamase producing hospital based infections.

The main aim of this study includes to know the prevalence of MBL production in various gram negative isolates. To determine the antimicrobial susceptibility pattern of the culture isolates by Kirby Bauer (Disc Diffusion method) as per CLSI guidelines. And also evaluate different phenotypic methods to detect MBL production (Disc Potentiation test) as per CLSI guidelines.

\section{Materials and Methods}

Study was done over a period of one year from January 2014 to December 2014 in the Department of Microbiology at VIMS, Ballari. Samples from 100 patients admitted in Burn unit were included in the study. Wound swabs were taken; samples received were cultured on 5\% sheep Blood Agar and MacConkey Agar plates \& also inoculated in BHI broth. Both plates and broth were incubated at $37^{\circ} \mathrm{C}$ for $24 \mathrm{hrs}$. Bacterial isolates were identified according to standard methods and tested against various antibiotics by Kirby Bauer disc diffusion method and zone diameters were interpreted according to the CLSI guidelines. MRSA was identified using disc diffusion method.0.5 McFarland standard suspension of organism in broth was prepared and inoculated in Mueller Hinton Agar (MHA) plate with $2 \% \mathrm{NaCl} .1 \mu \mathrm{g}$ oxacillin disc 
and $30 \mu \mathrm{g}$ cefoxitin disc (HiMedia) were applied over the inoculated media and incubated at $35^{\circ} \mathrm{C}$ for $24 \mathrm{hrs}$. Any colony or light film of growth around oxacillin and cefoxitin disc examined under transmitted light was considered MRSA.

The susceptibility to antipseudomonal drugs was done on Mueller Hinton agar by disc diffusion method in accordance with National Committee for Clinical Laboratory Standards (NCCLS) standards incorporating standard strain of $P$. aeruginosa (ATCC 27853). The antibiotics tested were gentamycin, amikacin, piperacillin, ciprofloxacin, ceftazidime, piperacillin-tazobactam, aztreonam (Hi-media Laboratories, Mumbai) and imipenem EDTA, extra pure (Hi-media Laboratories, Mumbai) powder was used for screening MBL production. Strains resistant to carbapenems were screened for Metallo beta lactamase (MBL) production by using two different tests:

\section{Imipenem (Imp)-EDTA combined disc Test (CDST)}

Performed as described by Yong et al., Test organisms were inoculated on to plates with Mueller Hinton agar as recommended by the CLSI. A 0.5 M EDTA solution was prepared by dissolving $18.61 \mathrm{~g}$ in $100 \mathrm{ml}$ of distilled water and adjusting $\mathrm{pH}$ to 8.0 by using $\mathrm{NaOH}$. The mixture was sterilized by autoclaving.

Two $10 \mu \mathrm{g}$ imipenem disks (Becton Dickinson) were placed on the plate, and appropriate amounts of $10 \mu \mathrm{L}$ of EDTA solution were added to one of them to obtain the desired concentration $(750 \mu \mathrm{g})$. The inhibition zones of the Imipenem and Imipenem-EDTA disks were compared after 16 to 18 hours of incubation in air at $35^{\circ} \mathrm{C}$. In the combined disc test, if the increase in inhibition zone with the Imipenem and EDTA disc was $\geq 7 \mathrm{~mm}$ than the Imipenem disc alone, it was considered as MBL positive.
IMP-EDTA double disk synergy test (DDST)/Modified-EDTA disc synergy test (MEDST)

Performed as described by Lee et al., ${ }^{[6]}$ Test organisms were inoculated on to plates with Mueller Hinton agar as recommended by the CLSI. An Imipenem $(10 \mu \mathrm{g})$ disc was placed $20 \mathrm{~mm}$ centre to centre from a blank disc containing $10 \mu \mathrm{L}$ of $0.5 \mathrm{M}$ EDTA (750 $\mu \mathrm{g})$. Enhancement of the zone of inhibition in the area between Imipenem and the EDTA disc is $\geq 5 \mathrm{~mm}$.

\section{Statistical analysis}

The statistical analysis is done using appropriate formula.

\section{Results and Discussion}

The prospective study conducted in the department of microbiology VIMS, Ballari showed the following findings: A total of 116 isolates were obtained from wound swabs collected from patients (100) admitted during the study period. Out of them $52 \%$ were female and $48 \%$ were male. About $60 \%$ of the patients were in the age group of 21-40 years and $70 \%$ of them had second degree burns. Out of 116 isolates, 82 were gram negative $(70.69 \%)$ and 34 were gram positive $(29.3 \%)$. Out of 100 wound swabs collected, 84 yielded single isolate and 16 yielded multiple isolates. The surface swabbing culture showed Pseudomonas aeruginosa (23\%) was the commonest organism from the burn wounds followed by Staphylococcus aureus (22\%), E. coli (14\%), K. pneumoniae (9\%), K. oxytoca (8\%), CONS(7\%), P. mirabilis (5\%), C.freundii (4\%), C. koseri (3\%), A. baumanii $(2 \%)$ and Non-fermenting GNB (1\%). Out of 82 gram negative isolates, 17 showed resistance to imipenem on antimicrobial susceptibility testing as done by Kirby Bauer Disk Diffusion method, following which they were subjected to disk potentiation tests (CDST and DDST)out of which only 10 
(12\%) were confirmed to be MBL producers. Isolation of MRSA was 56\% (14 out of 25), but all the strains were susceptible to glycopeptides and Linezolid.

Prevalence of MBL production was highest in A.baumanii (100\%) followed by P.aeruginosa $(21 \%)$.Total resistance pattern was noted in Ampicillin, Gentamicin, Ciprofloxacin and Ceftriaxone in case of MBL positive A.baumanii isolates. Pseudomonas aeruginosa was mostly sensitive to Piperacillin/Tazobactam, Gentamicin, Amikacin, Tobramycin, less sensitive to Ceftriaxone, Cefotaxime. Highest resistance was seen to ampicillin and ciprofloxacin (Chart 1). All the $10 \mathrm{MBL}$ positive isolates were sensitive to polymyxinB.

MBL-producing gram-negative organisms have now been reported in many geographic regions. Their ability to rapidly disseminate within an institution and lead to poor outcomes when infection ensues is a concern, and therefore, early laboratory detection is of great clinical importance ${ }^{7,8}$ Unfortunately, the phenotypic appearance of MBL-carrying organisms varies depending on the bacterial host, with increasing reports of carbapenemsusceptible isolates, primarily Enterobacteriaceae such as Klebsiella spp. and E. coli. ${ }^{9}$ Such organisms often carry hidden MBL genes, whereby the microbiologist and the clinician remain unaware of their presence within an institution. Such a scenario creates the potential for untoward clinical and infection control consequences and is by no means unique to MBLs. These isolates participate in horizontal MBL gene transfer with other gram-negative pathogens and contribute significantly to MBL-related outbreaks. Since there are no standard guidelines for detection of MBL, different studies have reported the use of different methods. PCR analysis is the gold standard method for the detection of MBL production, but it is not feasible in routine microbiology laboratory. Out of 116 isolates, 34 samples (29.31\%) were gram positive and $82(70.69 \%)$ were gram negative organisms. This is in concordance with two different studies conducted by Shobha Chamania et al., ${ }^{10}$ and Manjula Mehta et al., ${ }^{11}$ where the highest incidence of isolation of organisms from burn wounds was Gram negative organisms $(75 \%$ and $65 \%$ respectively), followed by Gram positive organisms. Out of 116 isolates, 84 samples yielded single isolates and 16 yielded multiple isolates. The predominant organism isolated from swab culture was Pseudomonas aeruginosa (23\%), followed by Staphylococcus aureus (22\%), E.coli (14\%), K.pneumoniae (9\%), K.oxytoca (8\%), CONS (7\%), Proteus mirabilis (5\%), C.freundii (4\%) and C.koseri (3\%). Out of 116 isolates, 2 were of Acinetobacter baumanii and one was identified as Non-fermenting GNB. Only 3 samples out of 100 yielded no growth on culture. This correlates to the Indian studies done by Manjula Mehta et $a l^{11}$, ShobhaChamania et $a l^{10}$, Sarita Mohapatra et $\mathrm{al}^{12}$ in which Pseudomonas aeruginosa forms the predominating organism $(52 \%, 43 \%$, and $31 \%$ and respectively) infecting the burn wounds. The ubiquitous presence of Pseudomonas aeruginosa in the hospital environment, intrinsic and acquired resistance to many antimicrobials, makes it a common pathogen in burn wound infections.

The antibiogram of Pseudomonas aeruginosa showed sensitivity to Piperacillin/Tazobactam (78\%), Gentamicin (57\%), Amikacin (76\%), Tobramycin $(57 \%)$. Total resistance pattern was noted in Ampicillin and Ciprofloxacin which coincides with the study done in Baghdad by Iman A Hussein et al., ${ }^{13}$. The resistance is high for third generation cephalosporins like Ceftriaxone (61\%), Ceftazidime (40\%) and Cefotaxime (53\%).This once again matches with the finding by Iman A Hussein et al., ${ }^{13}$ (Chart 24; Picture 1 and 2). 
Chart.1 Antimicrobial susceptibility of Pseudomonas aeruginosa

\section{AMS of P.aeruginosa}

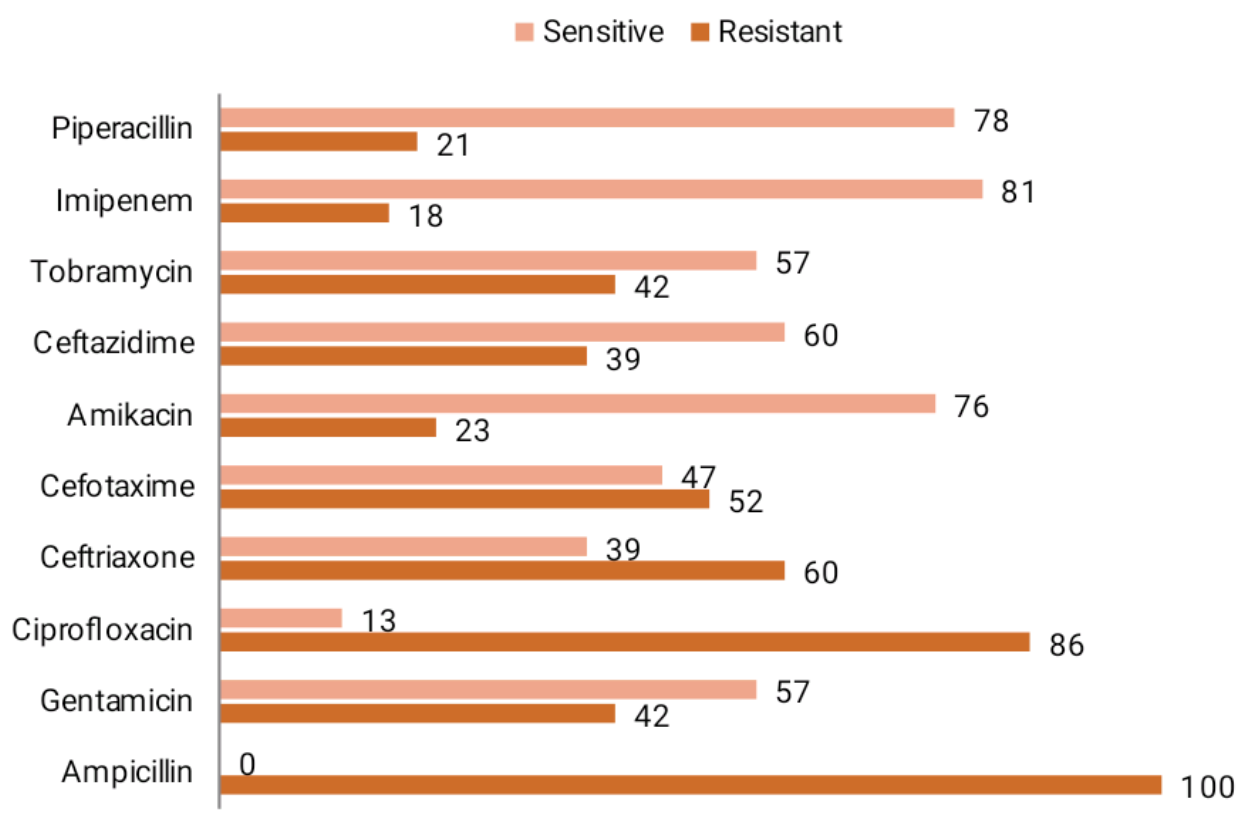

Chart.2 Antimicrobial susceptibility of Acinetobacter baumanii

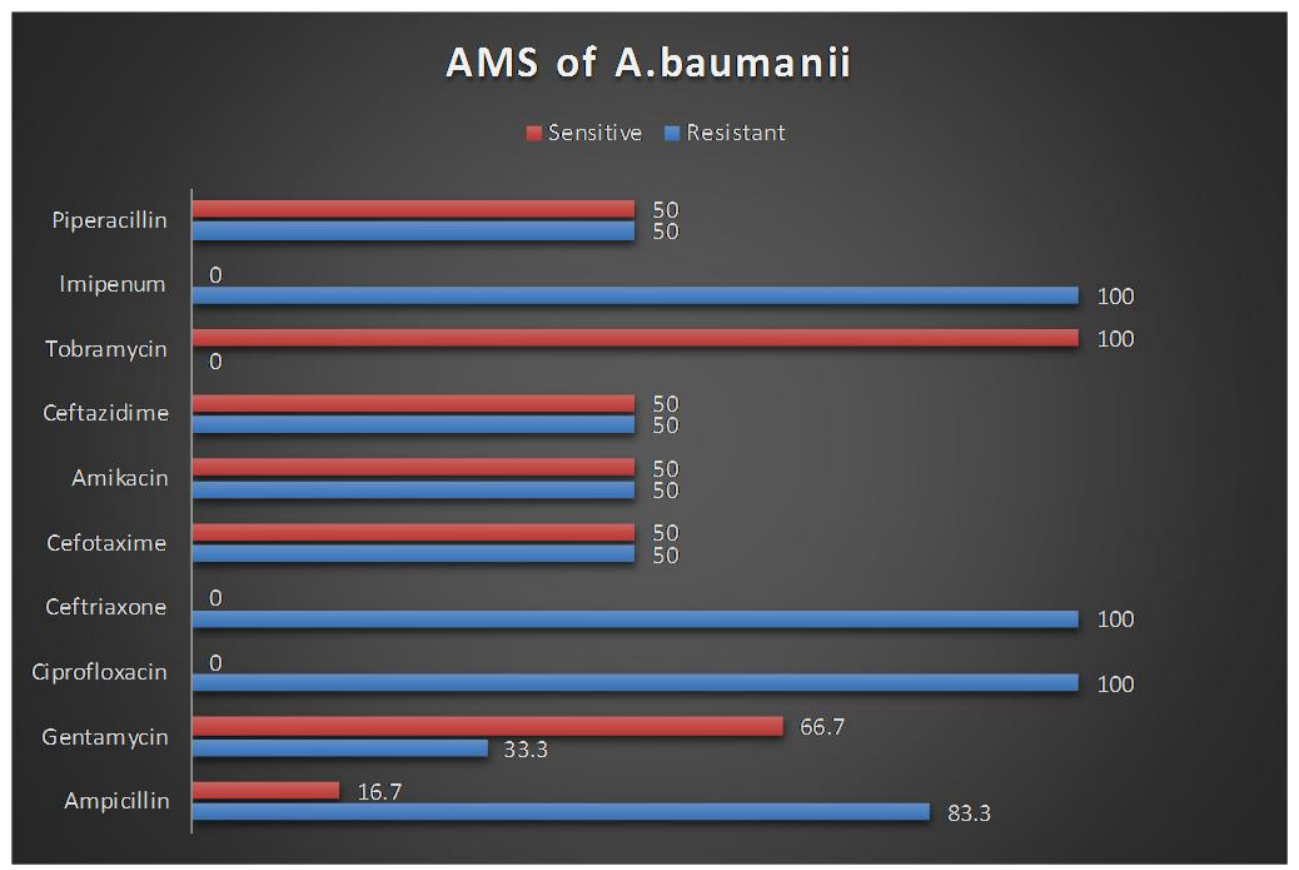


Chart.3 Shows multi drug resistant organisms isolated in the study (Total MDR=25)

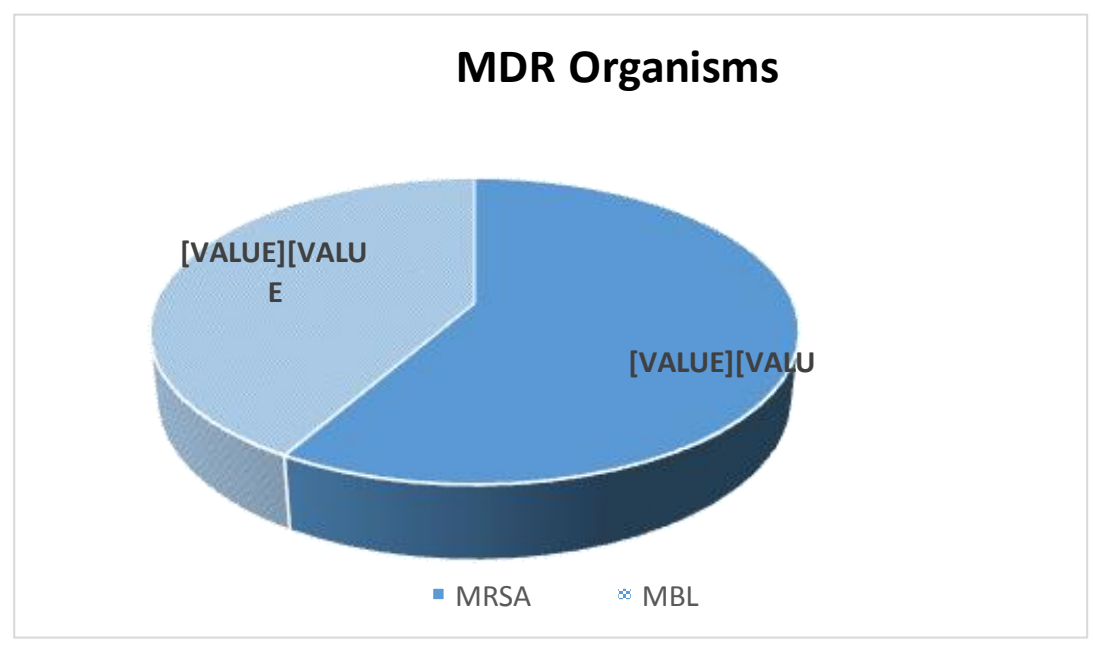

Chart.4 Shows number of Imipenem resistant and metallobeta lactamase +ve Gram negative Isolates

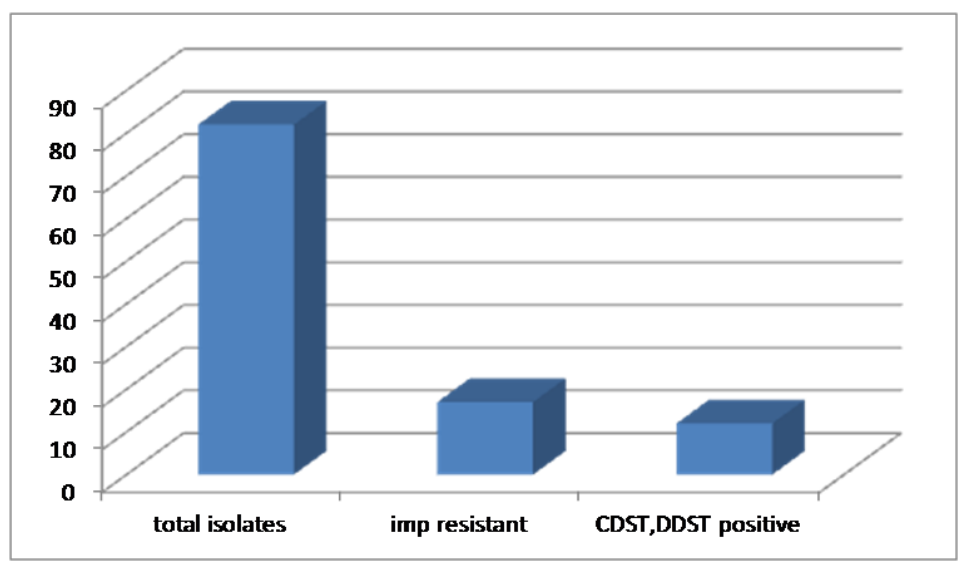

Pic.1 IMP-EDTA double disk synergy test (DDST)/Modified-EDTA disc synergy test (MEDST)

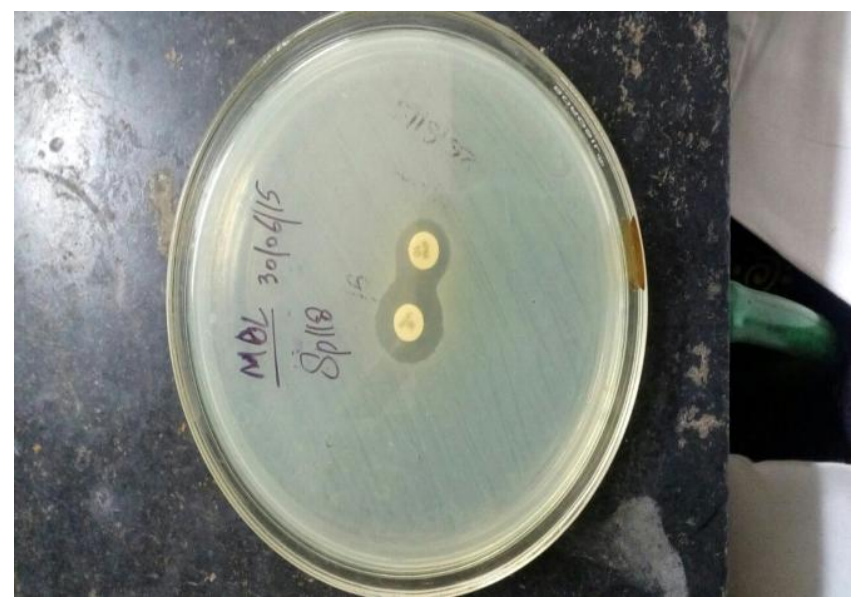




\section{Pic.2 Imipenem (IMP)-EDTA combined disc test(CDST)}

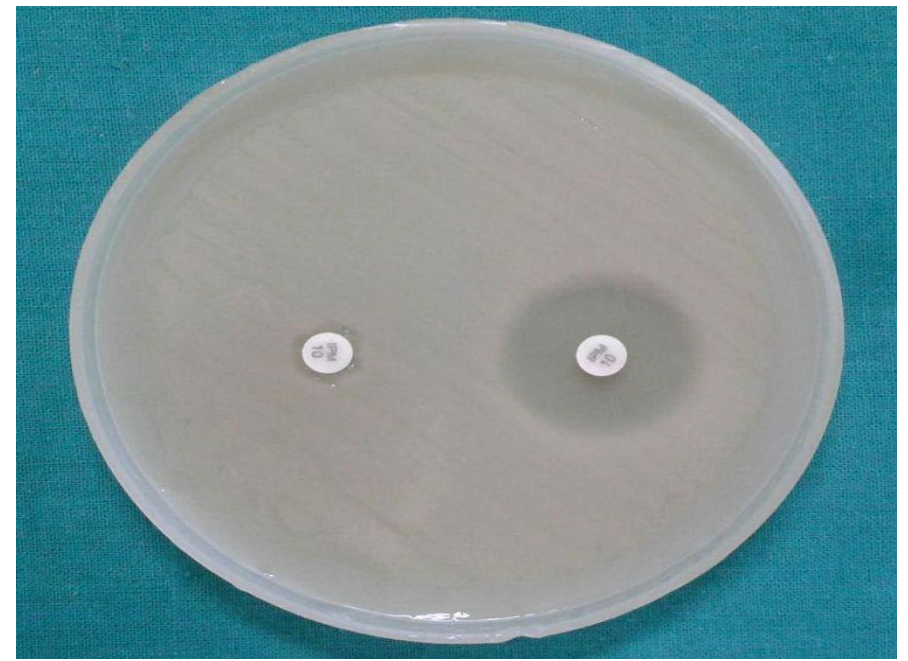

Out of 82 gram negative isolates, 17 showed resistance to imipenem on antimicrobial susceptibility testing as done by Kirby bauer Disk Diffusion method, following which they were subjected to disk potentiation tests(CDST and DDST) out of which only 10 $(12 \%)$ were confirmed to be MBL producers. The CDST method confirmed MBL production in $58.8 \%$ of screening positive isolates for MBL. Another study conducted by Aaftab et al., ${ }^{17}$ also showed similar results where the CDST method confirmed $68 \%$ of screening test positive isolates. Acinetobacter baumannii was the predominant MBL producer followed by Pseudomonas aeruginosa. This is in accordance with another study from India. However, all these isolates were sensitive to Polymyxin B. All the MBL producing strains were isolated from inpatients thus pointing to the fact that MBLs are largely a problem of hospitalized patients who share numerous risk factors. It has been claimed that polymyxins are not as toxic as previously thought ${ }^{14}$. However, they should not be used in monotherapy. A combination therapy must be preferred. An aminoglycoside or a fluoroquinolone molecule that may have retained some activity against the isolate may be chosen substantiated by rapid determination of its
MIC levels for the isolate. In the present scenario use of polymyxin $B$ should be judicious as strains with reduced susceptibility have emerged..$^{15}$ in the absence of novel agents the spread of MBL producers may lead to therapeutic dead ends. The increasing incidence of multiple $\beta$ lactamase producing organisms all over the world holds even more significance because organisms frequently also possess resistance factors to other classes of antibiotics, notably the aminoglycosides and fluoroquinolones. A study conducted in North Karnataka found co production of MBL and AmpC in $4.85 \%$ of the isolates ${ }^{17}$. Various studies have documented the incidence of multiple beta lactamase production ranging from $1.33 \%$ to $42.75 \%^{[18-21]}$. The reason for wide range in incidences could be factors like different antibiotic usage pattern leading to gene mutations or difference in normal flora depending upon cultural, nutritional or ethnic variations in different populations. The major cause of high incidence of ESBL and Amp C coproduction is inappropriate use of extended spectrum cephalosporins. ${ }^{22}$ The early detection of MBL producing $P$. aeruginosa may avoid the future spread of these multidrug resistant strains. 
In conclusion, our study validates a simple and highly sensitive phenotypic method for the detection of MBL production in a broad range of host organisms. Such organisms, carrying hidden MBL genes, are a threat to infection control efforts and may result in poor clinical outcomes when carbapenems are used for treatment in those with serious infections. The method is simple to perform, and the materials used are cheap, nontoxic, and easily accessible, making it highly applicable to routine clinical laboratories. The drawbacks of the study are Small sample size. Thus, all diagnostic laboratories must now be on high alert for MBL-producing organisms as early detection is critical to prevent the consequences of this worrying resistance mechanism.

\section{References}

1. A. Hussien Khalid A. Habib Kifah A. Jassim. Bacterial Colonization of Burn Wounds, 9(4): 2012; 326.

2. Nordmann, P, Poirel L. Emerging carbapenemases in gram negative aerobes. Clin. Microbiol. Infect., 8:321333.(2002).

3. Hemalatha, V, Uma Sekar \& Vijaylakshmi Kamat Indian. Detection of metallobetalactamase producing Pseudomonas aeruginosa in hospitalized patients. Med Res 122; 2005:148-152.

4. Clare Franklin, Lisa Liolios, and Anton Y. Peleg. Phenotypic Detection of Carbapenem-Susceptible Metallo- Lactamase-Producing Gram-Negative Bacilli in the Clinical Laboratory . Journal of clinical microbiology.2006; 44(9):3139-3144.

5. Yesim Cetinkaya, Pamela Falk, C. Glen Mayhall. Vancomycin-Resistant Enterococci. Clinical Microbiology Reviews, Oct 2000, 13 (4) 686-707

6. Lee, L-H, AbMutalib N-S, Law JW-F, Wong SH and Letchumanan V (2018) discovery on antibiotic resistance patterns of Vibrio parahaemolyticus in Selangor reveals carbapenemase producing Vibrio parahaemolyticus in marine and freshwater fish. Front. Microbiol. 9:2513. doi: 10.3389/fmicb.2018.02513

7. P. Pandya, et al., Evaluation of various methods for detection of metallolactamase (MBL) production in Gram negative bacilli. Int $\mathrm{J}$ Biol Med Res. 2011; 2(3): 775-777.

8. Noyal, M, Menezes G, et al., Simple screening tests for detection of carbapenemases in clinical isolates of nonfermentative Gram-negative bacteria. Indian J Med Res. 2009; 129: 707-712

9. Lee, CS, et al., Treatment of carbapenemresistant gram-negative bacteria. Infect Chemother 2014; 46(3):149-164

10. Chamania, et al., Burn wound infections and problems. Indian Journal of Burns. 2012 ; 20(1) :18-22

11. Mehta, M, Dutta P, Gupta V. Bacterial isolates from burn wound infections and their antibiograms: A eight-year study. Indian J Plast Surg. 2007; 40:25-28.

12. Mohapatra, S, Deb M, Agrawal K, Chopra S, Gaind R. Bacteriological profile of patients and environmental samples in burn intensive care unit: A pilot study from a tertiary care hospital. Indian J Burns 2014; 22:62-6.

13. Hussein, IA, Habib KA, Jassim KA. Bacterial Colonization of Burn Wounds. Baghdad Science Journal. 2012; 9:62331.

14. Nikolaos Markou, Critical Care 2003 7:R78 https://doi.org/10.1186/cc2358

15. Landman, et al., Citywide emergence of Pseudomonas aeruginosa strains with reduced susceptibility to polymyxin $\mathrm{B}$

16. Article in Journal of Antimicrobial Chemotherapy 55(6):954-7 June 2005

17. Aaftab G.P, Sumaira Qayoom Beigh, AaliyaKousar. Prevalence and detection of co-existence of multiple $\beta$ lactamases 
in clinical isolates: A diagnostic challenge and a dangerous trend journal of Microbiology and related research. 2018; 4:1-9.

18. Haider, M, Rizvi M, Fatima N, Shukla I, Malik A. Necessity of detection of extended spectrum betalactamase, AmpC and metallo-beta-lactamasesin Gram negative bacteria isolated from clinical specimens. Muller J Med Sci Res 2014; 5:23-8.

19. Grover, N, Sahni BAK, Bhattacharya S. Therapeutic challenges of ESBLs and Amp $\mathrm{C}$ beta lactamase producers in a tertiary care center. Med JArmed forces India 2013; 69:4-10.

20. Rawat, V, Singhai, M, Verma, PK.
Detection of different $\beta$ lactamases and their co-existence by using various disc combination methods in clinical isolates of Enterobacteriaceae and Pseudomonas spp. J Lab Physicians 2013;5:21-5.

21. Chatterjee, SS, Karmacharya R, Madhup SK, Gautham, V, Das A, Ray P. High Prevalence of Co-expression of newer $\beta$ lactamases (ESBLs, AmpC $\beta$-lactamases and metallo ßlactamases) in Gram negative bacilli. Indian J Med Microbiol 2010; 28: 267-8.

22. Turner PJ. Extended-Spectrum $\beta$ Lactamases. Clinical Infectious Diseases 2005; 41(4): S273-5.

\section{How to cite this article:}

Sumaira Qayoom Beigh, G.P. Aaftab, M.S. Rashmi and Muzafar Amin. 2019. Rising Incidence of Metallo B-Lacatamse Producers in Burns Injury: A Ticking Time Bomb. Int.J.Curr.Microbiol.App.Sci. 8(04): 2045-2053. doi: https://doi.org/10.20546/ijcmas.2019.804.240 